Els.Torreele@protonmail.com Follow Els on Twitter: @ElsTorreele Cite this as: BM/2020;370:m3209 http://dx.doi.org/10.1136/bmi.m3209 Published: 18 August 2020

\section{The rush to create a covid-19 vaccine may do more harm than good}

\author{
The focus on speed risks damaging public confidence in vaccines
}

\section{Els Torreele researcher and advocate}

The global race for a covid-19 vaccine seems under way to break all speed records. But this focus on rapid development, fuelled by unprecedented political, financial, and populist pressures, risks missing the target of global access to effective vaccines that can curb the pandemic, while irreparably damaging public confidence.

Vaccines are critical public health interventions because they prevent disease at population level, both protecting vaccinated individuals and curbing community transmission. That important aim, however, occurs only if the approved vaccine works well-an outcome now being chipped away at in the race for first marketing approval. Whether in Russia, where authorities announced approval of a vaccine without accompanying data, or in the US, with "Operation Warp Speed," vaccine developers and health authorities are now favouring speed over robust evidence of effectiveness and public health impact, constructing a narrative that anything is better than nothing, and lowering the bar on what constitutes a safe, effective, and useful vaccine.

The World Health Organization published a target product profile for covid-19 vaccines that specified minimum characteristics to guide developers-including 50\% efficacy. ${ }^{1}$ These are only aspirational, however, and neither companies nor regulators are bound to follow them. Officials from both the US Food and Drug Administration (FDA) and the European Medicines Agency have already signalled that they would consider approving vaccines that only diminished severity of illness, rather than protected against infection.

Meanwhile, companies have suggested acceptable efficacy goals much lower than $50 \%$ and have forewarned that protection may be relatively short lived. Key vaccine characteristics that are known to be critical for successful rollout globally-such as immunisation schedule (ideally a single dose), temperature stability (ideally no refrigeration), potential for rapid scale-up of manufacturing, and low cost-are currently not being considered in the competition for marketing approval. WHO has proposed a trial protocol that aims to test and compare different vaccine candidates in a standardised way, which would allow the best to be prioritised. ${ }^{2}$ But companies are not obliged to have their vaccines tested this way and, for obvious reasons, prefer setting up their own trials comparing their products with a placebo, with self-defined success criteria that may be less stringent than those of WHO.

Proponents of the vaccine race say that first generation vaccines "may not be as good as we would like" and that research and development will be needed over the next few years. ${ }^{3}$ Sensible as that may sound, a substandard first effort may not only harm people if corners are cut on safety, but also squander the opportunity to effectively channel the massive mobilisation of resources needed to curb the global pandemic and save millions of lives in a timely and equitable way, leaving crucial scientific questions unanswered and permanently altering the ability to develop and test better products.

One challenge is the inclusion in clinical trials of more vulnerable participants. It is common for initial vaccine trials to enrol healthy volunteers with homogeneous characteristics in terms of health, age, or ethnicity. Older adults with fragile health status, pregnant women, children, or those with comorbidities such as diabetes or tuberculosis, are typically excluded in early trials, as are otherwise vulnerable populations with little access to healthcare, such as poor communities, migrants, or refugees. Approving covid-19 vaccines untested among those groups could result in products that work best in those needing them least.

It is unlikely that one single vaccine will be equally effective in all populations. Instead, in order to effectively establish protection globally, we may need different types of vaccines that induce different kinds of immunity. For that to happen, a needs driven portfolio approach to development is needed, in which the scientific community collectively works towards advancing different vaccine profiles that can be assessed for their capacity to complement each other, rather than competing for the same niche. ${ }^{4}$

Hopes for better vaccines tomorrow depend on what we do today. The widespread use of a first generation vaccine with only $30 \%$ efficacy or a durability of only months will cripple the establishment of better vaccine candidates. In addition to scientific and methodological complexities having to compare with suboptimal vaccines, people's willingness to participate may wane, especially where populations financial resources and political will may not last to move second or third generation vaccines forward if initial-and unprecedented-investments fail to achieve significant health impact.

Voices from the vaccine establishment are raising concerns. Phil Krause, deputy director for vaccines for covid-19 vaccines, warned during a recent conference that a "weakly effective vaccine can do more harm than good." 5 The chief executive of Merck, Ken Frazer, has noted that "potential vaccines may not have the qualities needed to be rapidly deployed in large numbers" and that raising hopes for a vaccine before the end of the year are doing "a grave already distrust government motives. Importantly, at the FDA and member of the WHO working group 
disservice to the public." 6 These cautions, however, have been drowned out by media hype and political demands, such as Trump's pressure for a vaccine in $2020 .^{7}$

Another risk of speed over quality is the further erosion of trust in science by communities at risk. Covid-19 has already exposed the gap between science and practice, and the importance of working with communities to educate them about covid-19 risk, prevention, and treatments. Suboptimal experimental design or ethics practice lays the ground for suboptimal rollout hampered by distrust, vaccine refusal, and misinformation.

By setting the performance bar far lower in covid-19 vaccine development than what would otherwise be acceptable, we are also unwittingly redefining the concept of a vaccine-from a long term, effective preventive public health tool to what could amount to a population-wide suboptimal chronic treatment. This might be good for business but could prove fatal to global public health.

Competing interests: no competing interests

Not commissioned, not peer reviewed

1 WHO. WHO target product profiles for covid-19 vaccines. 29 April 2020. www.who.int/docs/default-source/blue-print/who-target-product-profiles-for-covid-19-vaccines.pdf.

2 WHO. Accelerating a safe and effective covid-19 vaccine. www.who.int/emergencies/diseases/novel-coronavirus-2019/global-research-on-novel-coronavirus-2019-ncov/accelerating-a-safe-andeffective-covid-19-vaccine.

3 Scientists don't expect a coronavirus vaccine to be nearly as protective as the measles shot. Fortune. 28 June 2020. https://fortune.com/2020/06/28/coronavirus-vaccine-protection-trialseffective.

4 Torreele E. Collective intelligence, not market competition, will deliver the best covid-19 vaccine Stat News. 10 June 2020. www.statnews.com/2020/06/10/collective-intelligence-not-marketcompetition-deliver-best-covid-19-vaccine.

5 WHO. Global scientific community unites to track progress on covid-19 R\&D, identifies new research priorities and critical gaps. 2 July 2020. www.who.int/news-room/feature-stories/detail/global-scientific-community-unites-to-track-progress-on-covid-19-r-d-identifies-new-researchpriorities-and-critical-gaps.

6 Grave disservice": Merck CEO warns against virus vaccine hopes. Al Jazeera. 16 July 2020. www.aljazeera.com/ajimpact/disservice-merck-ceo-warns-virus-vaccine-hopes200715040307280.html.

7 Abutaleb Y, DawseyJ. Trump pushing officials to speed up already ambitious coronavirus vaccine timeline. 17 June 2020. Washington Post. www.washingtonpost.com/health/2020/06/17/trumpcoronavirus-vaccine.

This article is made freely available for use in accordance with BMJ's website terms and conditions for the duration of the covid-19 pandemic or until otherwise determined by BMJ. You may use, download and print the article for any lawful, non-commercial purpose (including text and data mining) provided that all copyright notices and trade marks are retained. 